\title{
Some Classes of Function Spaces, Their Properties, and Applications
}

\author{
Józef Banaś, ${ }^{1}$ Janusz Matkowski, ${ }^{2}$ Nelson Merentes, ${ }^{3}$ Manuel Pinto, ${ }^{4}$ and Jose Luis Sanchez \\ ${ }^{1}$ Department of Mathematics, Rzeszów University of Technology, al. Powstańców Warszawy 8, 35-959 Rzeszów, Poland \\ ${ }^{2}$ Division of Functional Equations, Zielona Góra University, ul. Prof. Z. Szafrana 4a, 65-516 Zielona Góra, Poland \\ ${ }^{3}$ Department of Mathematics, Central University of Venezuela, Paseo Los Ilustres, Urb. Valle Abajo, Apartado Postal 20513, \\ Caracas 1020-A, Venezuela \\ ${ }^{4}$ Department of Mathematics, University of Chile, Casilla Postal 653, Santiago, Chile \\ Correspondence should be addressed to Józef Banaś; jbanas@prz.edu.pl
}

Received 10 October 2013; Accepted 10 October 2013

Copyright (C) 2013 Józef Banaś et al. This is an open access article distributed under the Creative Commons Attribution License, which permits unrestricted use, distribution, and reproduction in any medium, provided the original work is properly cited.

Function spaces create the basis of almost all investigations in several branches of mathematics such as functional analysis, nonlinear analysis, operator theory, and the theories of differential and integral equations, among others. Those spaces are frequently an object of the intensive study, in which properties of the mentioned function spaces are considered and described. First of all, such an approach is presented in functional analysis. But some topics of nonlinear analysis and operator theory are also closely related to the study of function spaces and their properties.

The second direction of investigations connected with the theory of function spaces depends on the application of that theory in the study of equations of various type, such as ordinary and partial differential equations, integral equations, functional differential, functional integral and functional equations, operator equations, and so forth. The study of the mentioned equations requires to place considerations in some function space. Consequently, all investigations associated with a considered equation are closely linked with a function space in which that equation is treated.

This special issue is devoted to discuss a lot of facts connected with the theory of function spaces. We study some properties of those spaces and we consider those aspects of functions spaces which are essential in miscellaneous applications. The papers published in this issue describe numerous spaces and indicate the applicability of those spaces in the study of several operator equations (differential, integral, and so on). We describe below the results contained in the papers published in this special issue.

At the beginning we present eight papers which are mainly devoted to describe some function spaces and their various properties.

In the paper of C. Tang a weighted Besov-type space and weighted Triebel-Lizorkin-type space are introduced. Moreover, the author obtained some characterization of those spaces expressed in terms of the so-called $\varphi$-transforms.

The paper of G. Hu is dedicated to some topics of homogeneous Triebel-Lizorkin spaces with full range of parameters. These spaces are introduced on stratified Lie groups in terms of Littlewood-Paley-type decomposition. The main result of the paper asserts that the scale of the considered TriebelLizorkin spaces is independent of the choice of LittlewoodPaley-type decomposition and the sub-Laplacian used for the construction of the decomposition.

The next paper written by $\mathrm{H}$. Mejjaoli discusses the Lorentz spaces associated with the Dunkl operators on the space $R_{4}$. Estimates of the Strichartz type for the DunklSchrödinger equations are obtained. The author considers also Sobolev inequalities between the homogeneous DunklBesov spaces and Lorentz spaces. C. Duyar and O. Ŏgur introduced in their paper a new space of double sequences related to $p$-absolutely convergent double sequence space, where $1 \neg p<1$. The main tools used in the construction of that space are an Orlicz function and an infinite double 
matrix. Some properties of the introduced space are established. For example, it is proved that the mentioned space is a paranormed space with a suitably defined paranorm.

The paper of M. Castillo et al. is concerning the space of functions with bounded $\kappa \varphi$-variation in the sense of RieszKorenblum. It is shown that the mentioned space creates a Banach space with an appropriate norm. The main result asserts that any uniformly bounded composition operator on that space satisfies the so-called Matkowski weak condition.

The paper of L. A. G. Méndez et al. contains a few results concerning the completion of the space of functions which are Henstock-Kurzweil integrable. The authors consider that space with the so-called Alexiewicz norm. Moreover, it is shown that this space has several other properties expressed in terms of Radon-Pettis property, Radon-Riesz property, and isometrical isomorphism, among others.

P. Chaoha, S. Iampiboonvatana, and J. Intrakul discuss in their paper the concept of the virtual stability of continuous self-mappings. That concept is generalized to arbitrary selfmappings. The structure of sequences connected with uniformly virtually stable self-mappings is studied. A necessary and sufficient condition for a uniform virtual stability of the space of the mentioned sequences is also obtained. The last paper belonging to the above announced group is due to $\mathrm{Z}$. $M$. Zheng et al. In that paper the author showed that the space of continuous periodic functions is the set of first category in the space of almost periodic functions. Moreover, it is shown that the space of almost periodic functions is the set of first category in the space of almost automorphic functions.

Next part of the papers which we would like to describe is composed of six papers. Those papers discuss various aspects of function spaces which are connected with some other topics expressed via operators, polynomials, interpolation, and so forth. Moreover, some applications of the discussed function spaces are also indicated. We start with the paper of A. Portilla et al. which describes asymptotic behaviour of extremal polynomials (with complex coefficients and equipped with a nondiagonal Sobolev norm) and the location of zeros of those polynomials under some hypotheses imposed on the diagonal matrix which is obtained via the unitary factorization.

Basic properties of nonlinear Kato class are established in the paper of R. E. Castillo and J. C. Ramos Fernández. The authors showed the strong continuation property of the eigenfunctions for the $p$-Laplacian operator defined on some Kato class.

Schrödinger operators are the main topic discussed in the paper of Y. Liu et al. It is shown, among others, that commutators of higher order associated with some Schrödinger operators are bounded if those operators act between a Hardy space and a Lebesgue space with the weak topology.

The paper of B. Sroysang concerns with some inequalities of Hermite-Hadamard type. The author proved also some integral inequalities involving several functions. C. Arteaga and I. Marrero discuss a function space arising in the theory of interpolation by Hankel translates of a basis function. The main result ensures that some Zemanian spaces are dense in the mentioned function space. Several other nontrivial results are obtained in this paper.
The paper of J. Banas and R. Nalepa discusses the function space consisting of functions having increments tempered by a given modulus of continuity. Such a space is a generalization of the classical spaces of Lipschitz or Hölder continuous functions. A criterion for relative compactness in that space is established. To show the applicability of that criterion, the author proved the solvability of certain quadratic integral equation of Fredholm type in the space of Hölder continuous functions.

Now, we describe the papers contained in this spacial issue which contain mainly applications of function spaces in the study of various operator equations. The first paper in this direction, written by M. Möller and B. Zinsou, contains considerations connected with a boundary value problem for a fourth order ordinary differential equation depending on an eigenvalue parameter. Moreover, the formulated boundary conditions depend linearly upon that parameter. The localization of eigenvalues is investigated and the first four terms of the asymptotic expansion of the eigenvalues are found. The considerations of the paper are placed in some Sobolev space.

In the paper of A. Farajzadeh and Salahuddin the study of the sensitivity analysis for nonlinear set valued variational equations is conducted. The resolvent operator technique is the main tool applied in that paper. The authors obtained a lot of results in the paper in question.

$\mathrm{S}$. $\mathrm{Ru}$ and J. Chen described in their paper a new class of function spaces with the help of frequency-uniform decomposition of a Lebesgue space. The authors considered also the Cauchy problem for Ginzburg-Landau equation in the mentioned function spaces.

Using classical Schauder-Tychonoff fixed point principle M. Gil and S. Wędrychowicz study solutions of the time-dependent Ginzburg-Landau equation. Moreover, they characterized those solutions in terms of local and global attractivity. Investigations of the paper are conducted in some Fréchet and Sobolev spaces.

The uniqueness of strong solutions of the Cauchy problem for the Camassa-Holm equation is studied in the paper of $\mathrm{M}$. Wu and C. Lai. That equation is considered in a suitable Sobolev space. The authors gave a new proof of the mentioned uniqueness result with the help of several auxiliary lemmas.

In the paper of I. Bachar et al. an existence theorem for a competitive fractional system is proved. The main tool used in the proof is the Schauder fixed point theorem. Apart of the existence of solutions the authors characterized those solutions in terms of global behaviour. The considerations are located in the classical space $C_{1,1}(D)$, where $D$ is a bounded subset of $R_{n}$.

A boundary value problem for a class of sequential integrodifferential equations of fractional order is considered by B. Ahmad and J. J. Nieto. The authors proved the existence result concerning the mentioned equations using the standard tools of the fixed point theory. The considerations are conducted in the classical Banach space consisting of real functions defined and continuous on a given segment $[a, b]$ and endowed with supremum norm.

The authors of the papers included in this special issue represent fourteen countries: China, Colombia, India, Iran, Japan, Mexico, Poland, Saudi Arabia, South Africa, Spain, 
Thailand, Turkey, USA, and Venezuela. Those countries are placed on five continents: Africa, North and South America, Asia, and Europe. This shows that this special issue includes the outcomes of the research work conducted over the whole world.

\section{Acknowledgments}

The guest editors of this special issue would like to express their immense gratitude to the authors of all papers submitted for considerations. We hope that the results included in this issue will be an inspiration for researchers working in the theory of function spaces and nonlinear analysis.

Józef Banaś Janusz Matkowski Nelson Merentes Manuel Pinto Jose Luis Sanchez 


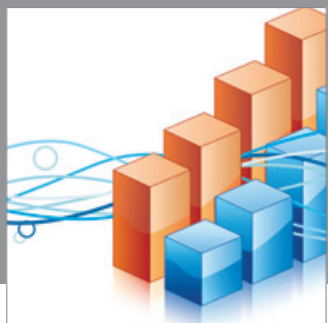

Advances in

Operations Research

mansans

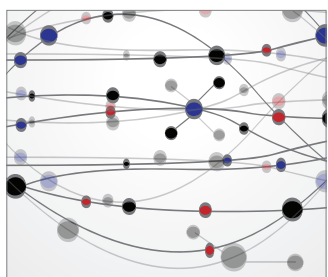

The Scientific World Journal
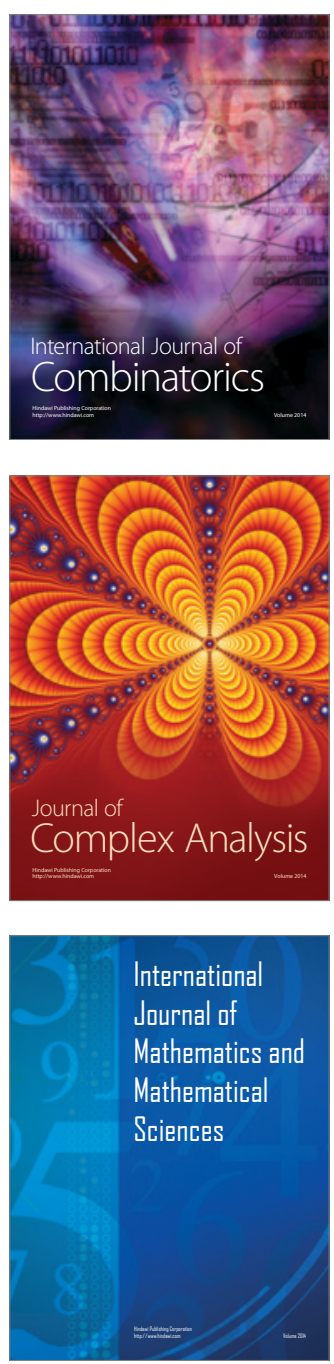
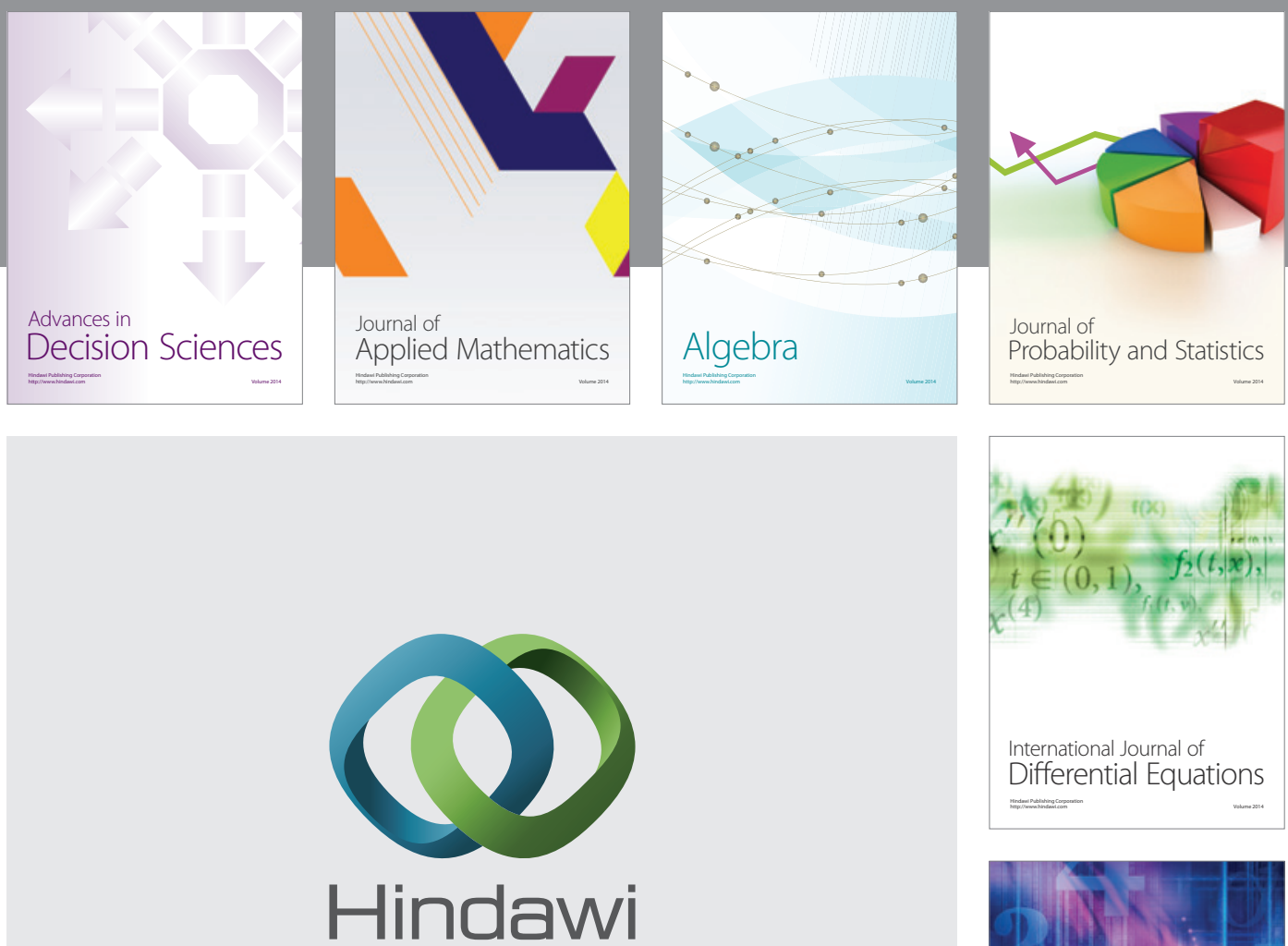

Submit your manuscripts at http://www.hindawi.com
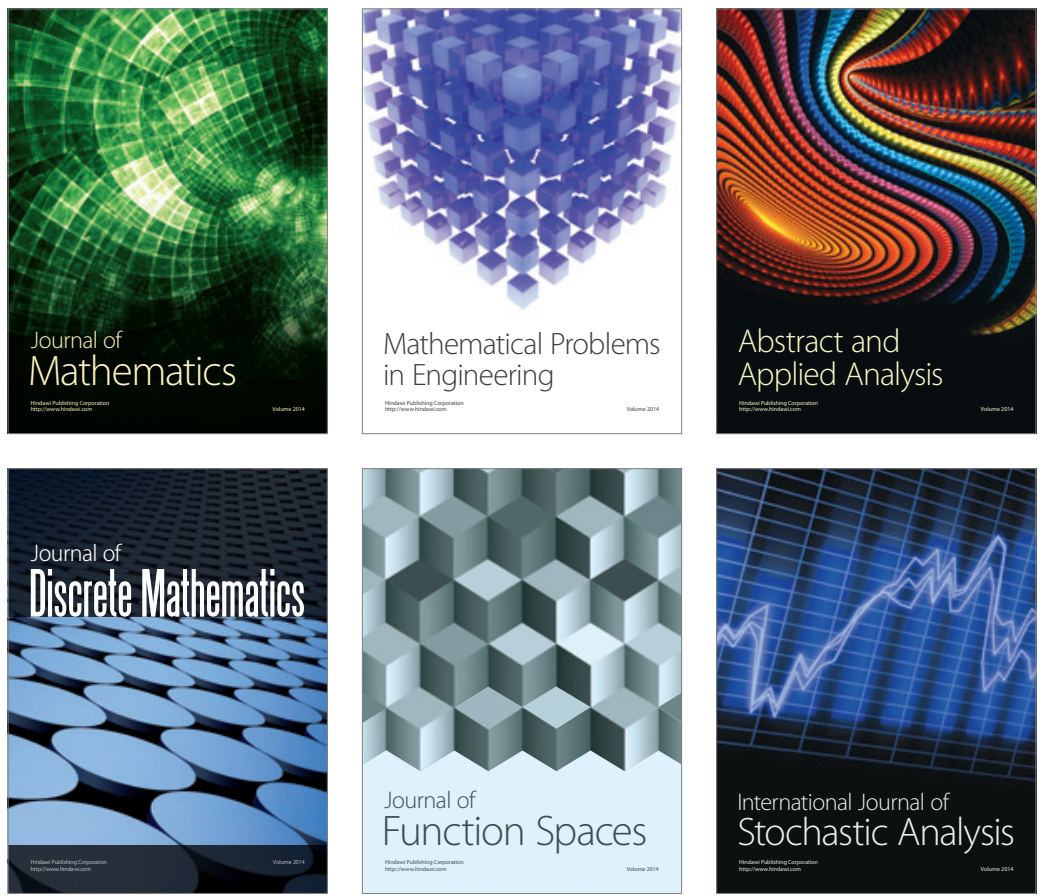

Journal of

Function Spaces

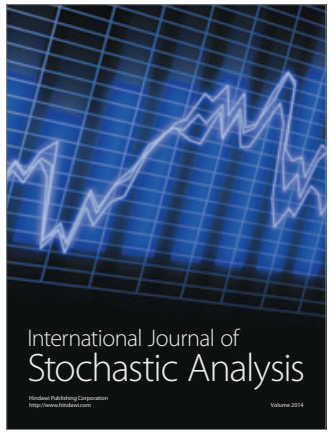

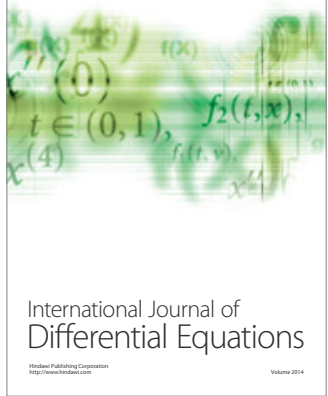
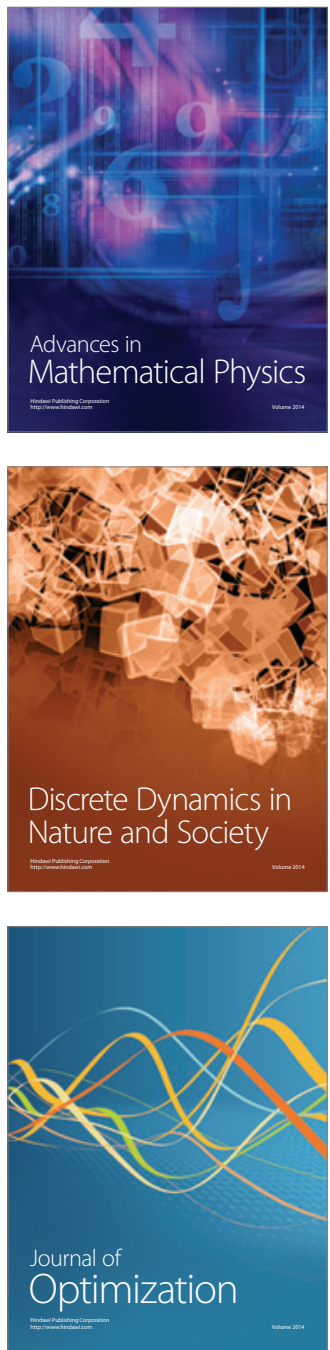